\title{
Global impact of COVID 19 on 112 Emergency System detailed for National 112 Emergency System of Romania
}

\author{
Mihail Bărănescu ${ }^{1,}$, , Ionel-Sorinel Vasilca ${ }^{1}$, MădălinaTabarcia ${ }^{1}$, and Madlena Nen $^{2}$ \\ ${ }^{1}$ The Bucharest University of Economic Studies, Piața Romană 6, Bucharest, Romania \\ ${ }^{2}$ The Bucharest Military Technical Academy Ferdinand I, Bulevardul George Coşbuc 39-49, \\ Bucharest, Romania
}

\begin{abstract}
.
Research background: During COVID19 crisis, in order to limit and control the multiplication of infections with the new virus, the Romanian Government forced restrictions and established fines to those not staying in line with the rules. The rules had been applied not only to citizens but also to private companies and state authorities. Due to these legal constraints taken during the declared state of emergency and the next state of alert, the appropriate attitude effects occurred in terms of behaviour of individuals and companies.

Purpose of the article: One of the positive side effects of the measures taken during the state of emergency and the state of alert, is a decrease of non-real emergency calls in public safety answering points, small in value but important for dealing with real emergencies. Moreover, the incoming calls related to some chronic non-urgent medical affection decreases.

Methods: We will study and make comparisons in the number of calls in 112 Emergency System to analyse the quantitative impact relative to COVID period versus a previous not impacted period of time because of COVID.

Findings \& Value added: The impact of the pandemic on the 112 Emergency System has been a major one. We observed the need to redefine procedures and to make operational a new resilience plan, by defining a specific calling number where people in need to report incidents specifically related to COVID. We also observed some changes in caller behaviour that occurred to those calling the emergency 112 number. However, the last not the least we have observed a real interest of people calling to the specific number dedicated to COVID with different contexts, concerns and clarifications needed to them in order to adopt a more conscious and responsible attitude.
\end{abstract}

Keywords: emergency calls; pandemic; COVID-19; non-emergency calls; emergency $112 \mathrm{~s}$

JEL Classification: $C 01 ; 118 ; 115 ; C 83 ; C 80$

\footnotetext{
*Corresponding author: baranescumihail@yahoo.com
} 


\section{Introduction}

The COVID-19 pandemic, also known as the coronavirus pandemic, is an ongoing pandemic of coronavirus disease 2019. More than 30 million cases have been reported worldwide.

"The COVID-19 pandemic confronted the world with a rapid, unexpected and far reaching global crisis. While it began as a healthcare emergency, it very rapidly became clear that the pandemic would have unprecedented political, economic and social consequences. " [1]

"The COVID-19 pandemic has resulted in over 4.3 million confirmed cases and over 290,000 deaths globally. It has also sparked fears of an impending economic crisis and recession. Social distancing, self-isolation and travel restrictions have led to a reduced workforce across all economic sectors and caused many jobs to be lost." [2]

"Public health crises test the robustness of institutions, and the current COVID-19 pandemic is no exception. The spread of the virus and subsequent hospitalizations and deaths have laid bare the fragility of governments' capacities to protect their citizens, and it has strained otherwise normally functioning institutions (...).“ [3]

The first case of a new coronavirus, COVID-19, was reported in Romania on 26th of February 2020. The evolution of the epidemic has reached a key moment in which the number of cases curve has not flattened, nor has there been a sudden epidemiological peak, due to the fact that the authorities took measures shortly after detecting the first case and the decisions taken were clearly communicated to citizens. Lessons learned from cooperation with other European countries has been also really helpful.

At that time, in the 112 emergency call centres we faced the situation of receiving a high volume of calls due to the COVID-19 outbreak. "Initially, the number of calls increased when COVID-19 was considered a pandemic by the World Health Organization (WHO) and as the number of infected people grew. The number of calls also increased after some countries announced the state of emergency. As the entire world focuses its efforts into facing the COVID-19 challenge, The European Emergency Number Association EENA has gathered information on the increase in number of emergency and the strategies followed by different countries" [4].

In Romania, some of the most common strategies to manage the COVID-19 outbreak included an increase of number of operator staff to decrease the queue at the entrance in the 112 system, making the public announcement for people to call the emergency number only in case of emergencies, and in parallel creating specific hotline reversed-paid number to answer to specific questions and concerns of people about COVID-19." Citizens were advised to consider only the accurate information checked and double-checked by official sources and to call for recommendations and other information on the dedicated COVID-19 line while letting 112 lines available for real emergencies. In the same time, to support the Romanian people relocated abroad, they were able to request for official information about preventing and combating the virus at a line specially dedicated to them" [5].

"The viral contagion has also created a social contagion in terms of technology adoption behaviours." [6]

One can see that the primary reason for an overall decrease in call volume is that people were simply staying at home, with less situations to be at danger, such as those that would occur when people are driving back and forth to work or to school or when congregating in public places.

To optimize the handling of calls made to the 112 single emergency number, the Special Telecommunications Service, together with the Department for Emergency Situations of Ministry of Interior and the Ministry of Health, updated the rules and public 
communications, in accordance with the evolution of cases, and also updated the 112 index for a better classification of calls based on the situations reported by citizens.

It is to note that, when the pandemic started, the 112 Emergency service received calls from people who returned in Romania or from those who had contacts with persons returning from affected countries by Sars-Cov-2 and that wanted to inform the authorities about such situations, and, subsequently, many people called to get additional information about the restrictions imposed or to notify about their non-compliance.

Regarding the procedure specific to call takers, and for a better discrimination of calls related to COVID-19, the STS operators analysed carefully each and every incoming call.

"We argue that a core role of communication is to transmit information that helps coworkers do their job better." [7]

"Thus, the calls during which people reported particular symptoms were directed to the healthcare agencies, the calls related to persons who did not comply with the selfisolation/quarantine established by authorities were directed to the Police, and when callers needed particular information, they were advised to call the support line Helpline at 0800.800 .358 ". [8]

"COVID-19 and the large scale social and economic shock which it bought has already profoundly transformed organizational cultures. Well known symbols of organizational life such as open plan workplaces filled with people wearing suits have been replaced by Perspex screens and personal protective equipment." [9]

Social distancing during the COVID-19 pandemic will save lives. The findings suggest that the thought of infecting vulnerable people or large numbers of people can motivate social distancing. This has implications for communications strategies. The study also demonstrates an effective way to identify outcome variables for rapid behavioural research on the COVID-19 response. [10]

It is to be mentioned that the Special Telecommunications Service joined the initiative launched by the Emotional Intelligence Research Association (EmIRA) and thanks to the support of Telekom Romania operator, made available the Psychological Counselling and Emotional Support Helpline to the people hospitalized, quarantined or in isolation, to the healthcare personnel under stress, and to all those affected by the COVID-19 pandemic. This helpline was operational starting 8th of May 2020, and it aims to assist the vulnerable persons in need during the current crisis. This line can be used also by the hospitalized, the isolated or the quarantined, by healthcare personnel, or by anyone, regardless of age, who feels fear or anxiety in relation to the circumstances generated by the coronavirus pandemic. "Callers are assured of the confidentiality of their conversations and identity, and their personal data is not to be further processed or stored in any way. Calls are answered every day, between 09:00 and 21:00h, as helpdesk operator being certified psychologists, specialized in offering counselling and support and in the management of stressful life events". [11]

"Though it is difficult to predict when or how the pandemic will recede, it is easy to appreciate that it affords important lessons for organizational learning. Many actions that organizations learn to take in response to a pandemic are meant to save lives" [12]

\section{Methods}

From the beginning of the state of emergency, the Special Telecommunications Service, as administrator of the 112 system, has been implementing the necessary measures to take all calls 24/7, even in overcrowded conditions. During the last couple of months, the 112 Service has not encountered any operational or technical difficulties while taking and processing the incoming calls. 


\subsection{State of emergency}

"Between March 16th and May 14th 2020, the STS operators received 1,558,679 incoming calls to 112 , of which 832,226 were emergencies, $5.07 \%$ of the calls being related to the new coronavirus.

Most of the calls regarding COVID-19 issues were made from Bucharest and the highest volume of calls made to 112 related to the coronavirus was registered during 12 and 30 of March 2020, when the daily average reached approximately 1,300 calls". [13]

During the declared state of emergency (March, 15th to May, 14th), the number of emergency calls generated to the 112 and the number of calls of non-emergency calls, which did not require the intervention of the specialized field agencies, were on a downward slope, compared to the normal periods of the previous year, as we can see in the chart below:

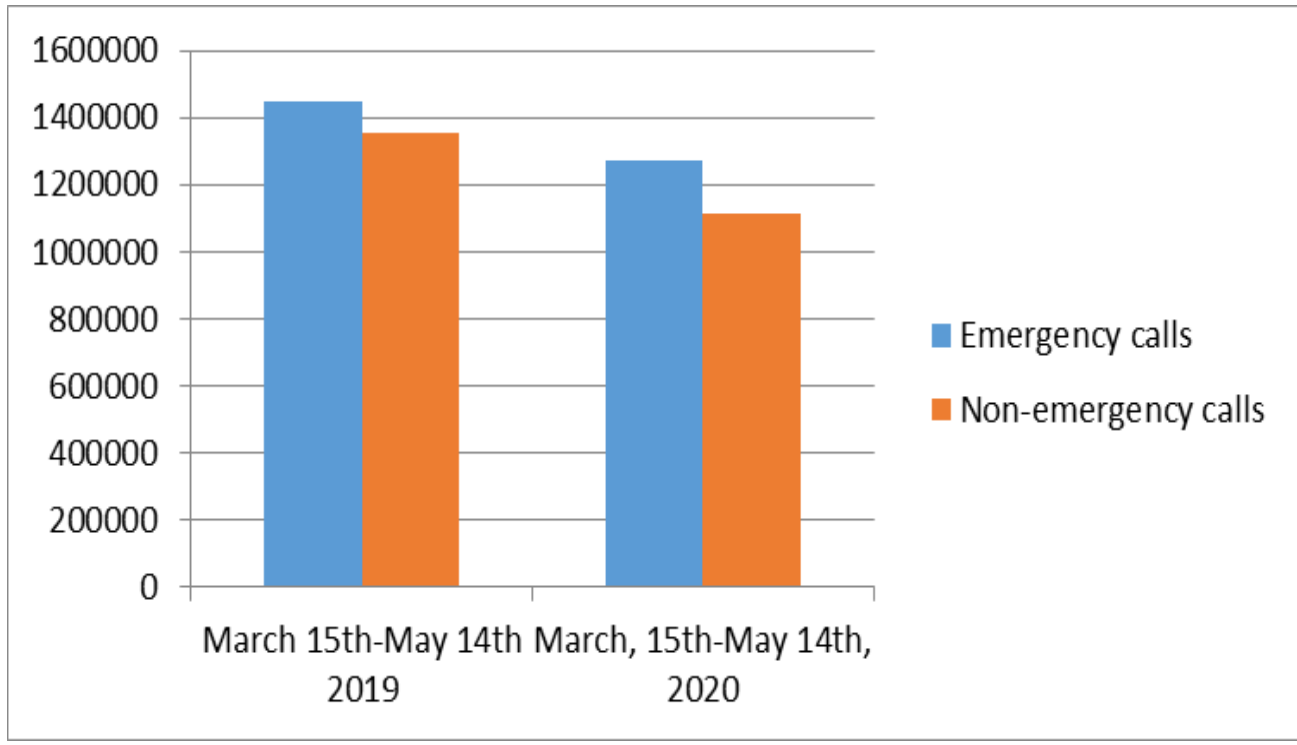

Fig. 2. 112 calls in state of emergency

\subsection{Alert State}

Similarly, due to alternative offered helpline number, there was a decrease in the number of calls received at the emergency number 112 compared to the same period of 2019, during the alert state (May,15th-July,31th), as follows: 


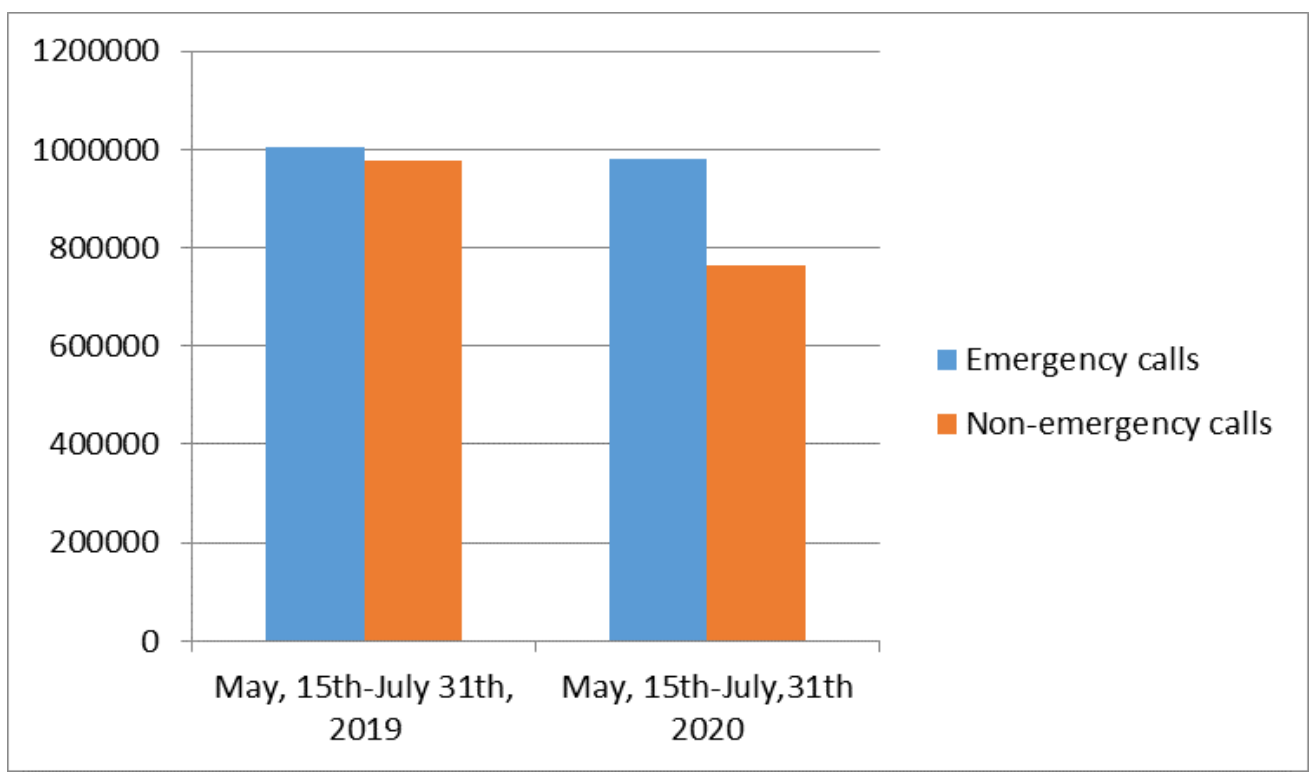

Fig. 2. 112 calls in alert state

The number of emergency calls related to medical issues also has been dropped but this fact is alarming because people thought that is not safe to go to a hospital, in other words they did not seek treatment being afraid of catching the virus in a hospital.

\subsection{Call number decreases impact}

The decrease of the number of non-emergency calls had a positive impact on the quality of service offered by the 112 system. In the context of the COVID-19 pandemic, 112 emergency operators took 70,338 calls by which citizens reported specific symptoms of the virus, the fact that they returned from outbreaks, came into contact with people confirmed positive or were aware of violations of the restrictions established by the authorities.

In a large percent of cases, people called the unique emergency number 112 to find out information about the new coronavirus, without having any real emergencies. "In this regard, the administrator of the emergency 112 system reminded them that the number TELVERDE - 0800.800.358, a telephone line specifically located to inform citizens about COVID-19, has been operationalized". [14]

It is noteworthy that, in this context, the number of non-emergency calls has decreased, which shows that citizens have understood the importance of responsibly calling the unique emergency number 112. The changes in the public's behaviour as a result of lockdown may have had some positive effects, such as fewer accidents occurring outside the home, as well as difficult time for those with a chronic disease, needed to stay at home but that should be seeking medical support for dialysis or other therapies that asked for periodic medical visit at the hospitals.

The Romanian Special Telecommunications Service announces that in the first three months of 2020, there were 2,539,700 calls to 112 reporting cases of domestic violence, $13 \%$ more than the same period last year, concluding that Romanians became more responsible with the onset of the pandemic. Although the phenomenon is growing, it is no longer being reported. The biggest problem is that victims can no longer talk on the phone because aggressors are next to them all the time and that of contracting the coronavirus by calling the ambulance to be taken to the hospital. 
Unrelated to COVID-19 pandemic, but with an impact on declining the number of nonemergency calls was the change in legislation adopted at the end of March, 2020, regarding the new penalties applied for abusive call of the 112 Emergency Service, which is now punishable by fines or by community work service hours, has also contributed to the decrease of the number of non-emergency calls.

"The current coronavirus (COVID-19) pandemic is again reminding us of the importance of using telehealth to deliver care service, especially as means of reducing the risk of cross-contamination caused by close contact. For telehealth to be effective as part of an emergency response, it first needs to become a routinely used part of our health system. Hence, it is time to step back and ask why telehealth is not mainstreamed. Strategies to ensure that telehealth is used regularly in acute, post-acute and emergency situations, alongside conventional service delivery methods, include flexible funding arrangements, training and accrediting our health workforce. Telehealth uptake also requires a significant change in management effort and the redesign of existing models of care. Implementing telehealth proactively rather than reactively is more likely to generate greater benefits in the long-term, and help with the everyday (and emergency) challenges in healthcare". [15]

"It is urgently advised and requested that all the persons should follow the preventive measures, managements and quarantine strictly without any religious discrepancy otherwise the situation may be the worst. Also, there is an urgent requirement to educate our new generation for science and technology to fight against any such disaster in future, if any." [16]

\section{Conclusions}

Unexpected global events, such as the Covid-19 pandemic can have significant effects on emergency services. The analysis of emergency and non-emergency calls in Romania since the declared state of emergency highlights a decrease in calls received during this period compared to the same period last year. Among the positive effects, there is a decrease in calls that did not represent emergencies. However, a similar decrease in urgent calls may just be citizens being reluctant calling the single emergency number, which may pose a substantial risk to them in the future, and these issues could remain unresolved.

It is important that everyone accept that it is very safe to call 112 , or to go to the hospital for acute medical problems, because hospitals have taken many steps to assure patient safety and medical staff is well prepared to safety intervene for serious medical issues.

The psychological effects of living in isolation can only be suspected in comparison with the effects of financial problems, which are truly well documented. During Covid-19 pandemic, cases of domestic violence have been also increased due to unemployment.

It is certain that in addition to the direct threat posed by the virus itself, the pandemic already has many extra-medical effects, which cause changes in the way we live our lives.

\section{References}

1. Muzio D., Doh J. (2020). Introduction to COVID-19 Commentaries. Journal of Management Studies, 57(8), 1725-1726.

2. Nicola M., Alsafi Z., Sohrabi C., Kerwan A., Al-Jabir A., Iosifidis C., Agha M., Agha R. (2020). The socio-economic implications of the coronavirus pandemic (COVID-19): A review. International Journal of Surgery, 78, 185-193.

3. King B., Carberry E. J. (2020). Movements, Societal Crisis, and Organizational Theory. Journal of Management Studies, 57(8), 1741-1745. 
4. European Emergency Number Association (2020, March). Data and strategies on emergency calls \& public warning during the Covid-19 outbreak. Retrieved from : https://eena.org/knowledge-hub/press-releases/data-strategies-emergency-calls-publicwarning-covid19/

5. Ministerul Afacerilor Interne (2020, March). Informare 20 martie 2020, ora 13:00. Retrieved from : http://www.dsu.mai.gov.ro/informare-20-martie-2020

6. Gerard G., Karim R. L., Phanish P. (2020). What has changed? The Impact of Covid Pandemic on the Technology and Innovation Management Research Agenda. Journal of Management Studies, 57(8), 1754-1758.

7. Battiston D., Blanes J., Kirchmaier T. (2017). Face-to-Face Communication in Organisations. The Review of Economic Studies.

8. The Special Telecommunications Service (2020, May). Statistics of the calls made to 112 during the state of emergency. Retrieved from : https://www.sts.ro/en/pressreleases/statistics-of-the-calls-made-to-112-during-the-state-of-emergency

9. Spicer, A. (2020). Organizational Culture and COVID-19. Journal of Management Studies, 57(8), 1737-1740.

10. Lunn, P. D., Timmons, S., Barjaková, M., Belton, C. A., Julienne, H., Lavin, C. Motivating social distancing during the Covid-19 pandemic: An online experiment. Social Science \& Medicine, 113478.

11. The Special Telecommunications Service (2020, May). 021.9696 - Psychological Counselling Helpline for People Affected by the Coronavirus Epidemic. Retrieved from : https://www.sts.ro/en/press-releases/021-9696-psychological-counsellinghelpline-for-people-affected-by-the-coronavirus-epidemic

12. Greve, H. R. (2020). Learning theory: The pandemic research challenge. Journal of Management Studies, 57(8), 1759-1762.

13. The Special Telecommunications Service (2020, July). Decrease in non-emergency calls to 112 . Retrieved from : https://www.sts.ro/en/press-releases/decrease-in-nonemergency-calls-to-112

14. Smith, A. C., Thomas, E., Snoswell, C. L., Haydon, H., Mehrotra, A., Clemensen, J., Caffery, L. J. (2020). Telehealth for global emergencies: Implications for coronavirus disease 2019 (COVID-19) Journal of telemedicine and telecare, 26(5), 309-313.

15. Imran A., Alharbi O. (2020). COVID-19: Disease, management, treatment, and social impact Science of the Total Environment, 728, 138861. 